\title{
Lack of Frank Agrammatism in the Nonfluent Agrammatic Variant of Primary Progressive Aphasia
}

\author{
Naida L. Graham a, b Carol Leonard ${ }^{c}$ David F. Tang-Waid, e \\ Sandra Black ${ }^{\mathrm{e}-\mathrm{g}}$ Tiffany W. Chow ${ }^{\mathrm{e}, \mathrm{g}, \mathrm{h}}$ Chris J.M. Scott ${ }^{f}$ \\ Alicia A. McNeely ${ }^{f}$ Mario Masellis ${ }^{f}$ Elizabeth Rochon ${ }^{a, b}$ \\ ${ }^{a}$ Department of Speech-Language Pathology, Faculty of Medicine, University of Toronto, \\ b Toronto Rehabilitation Institute, Toronto, Ont., ' Department of Audiology and Speech- \\ Language Pathology, University of Ottawa, Ottawa, Ont., dUniversity Health Network \\ Memory Clinic, Toronto Western Hospital, e Department of Medicine (Neurology), University \\ of Toronto, ${ }^{f}$ L.C. Campbell Cognitive Neurology Research Unit, Sunnybrook Health Sciences \\ Centre, ${ }^{9}$ Rotman Research Institute, and h Department of Psychiatry (Geriatric Psychiatry), \\ University of Toronto, Toronto, Ont., Canada
}

Key Words

Diagnostic criteria - Frontotemporal dementia - Apraxia of speech - Differential diagnosis

\begin{abstract}
Background/Aims: Frank agrammatism, defined as the omission and/or substitution of grammatical morphemes with associated grammatical errors, is variably reported in patients with nonfluent variant primary progressive aphasia (nfPPA). This study addressed whether frank agrammatism is typical in agrammatic nfPPA patients when this feature is not required for diagnosis. Method: We assessed grammatical production in 9 patients who satisfied current diagnostic criteria. Although the focus was agrammatism, motor speech skills were also evaluated to determine whether dysfluency arose primarily from apraxia of speech (AOS), instead of, or in addition to, agrammatism. Volumetric MRI analyses provided impartial imaging-supported diagnosis. Results: The majority of cases exhibited neither frank agrammatism nor AOS. Conclusion: There are nfPPA patients with imaging-supported diagnosis and preserved motor speech skills who do not exhibit frank agrammatism, and this may persist beyond the earliest stages of the illness. Because absence of frank agrammatism is a subsidiary diagnostic feature in the logopenic variant of PPA, this result has implications for differentiation of the nonfluent and logopenic variants, and indicates that PPA patients with nonfluent speech in the absence of frank agrammatism or AOS do not necessarily have the logopenic variant.




\section{Introduction}

Primary progressive aphasia (PPA) is a neurodegenerative syndrome in which language is the earliest and most severely affected aspect of cognitive functioning. In the nonfluent variant (nfPPA), speech is effortful and hesitant, resulting in a reduced rate of speaking. Recently published international consensus criteria [1] require that either one of two core features must be present for diagnosis of nfPPA: agrammatism in language production and/ or effortful speech with inconsistent speech sound errors and distortions, usually due to apraxia of speech (AOS). The logopenic variant (lvPPA) is characterized by impaired word retrieval, phonological errors in speech, and impaired repetition of sentences and phrases. In contrast with nfPPA, there should be sparing of motor speech skills and no frank agrammatism in lvPPA [1]. In some cases, however, the nonfluent variant may be difficult to distinguish from the logopenic variant because both may be associated with reduced speaking rate, hesitations, and other features of dysfluency [2-7].

A large body of work, based mainly upon patients with aphasia following stroke, has identified the main features of agrammatism in spoken language. Agrammatism is characterized by a tendency to omit and/or substitute both bound and free grammatical morphemes (resulting in syntactic errors), with associated reductions in proportion of verbs, and syntactic complexity [8-10]. In extreme cases, speech may be telegraphic. Although agrammatism is an established concept in aphasiology, the term can also be interpreted more loosely to mean any type of grammatical impairment. In the interests of clarity, we will therefore use the term 'frank agrammatism' to refer to the classical definition of agrammatism as outlined above.

Despite not being required for the diagnosis of nfPPA, the importance of agrammatism as a main deficit is supported by a number of key findings. In particular, frank agrammatism (of the type seen in Broca's aphasia following stroke) has been documented in nfPPA [2, 11-16]. Early reports highlighted the similarity between agrammatic (Broca's) aphasia following stroke and nfPPA [17-21]. The idea that the aphasia is comparable in these distinct pathologies persists, and indeed there are clear similarities. For example, quantitative analyses of connected speech in groups of nfPPA patients have shown that people with nfPPA produce more grammatical errors and/or fewer well-formed sentences than controls $[2,3,6$, $13,22-25]$. Note, however, that in some reports the presence of syntactic or agrammatic errors was a requirement for diagnosis of nfPPA [e.g., 24], while in other reports it was not specified whether this was required.

Although frank agrammatism occurs in nfPPA, there are findings demonstrating that it is not universal. For example, group studies have shown that patients with nfPPA may produce normal proportions of verbs in connected speech [2,13,25-27], a finding not expected in frank agrammatism; contrary results were, however, obtained by Ash et al. [24, 28] and by Wilson et al. [23], although in the latter report the significant effect was said to be 'driven' by 3 (of 14) patients. Frank agrammatism is also associated with reduced production of closed class words, but some studies have found no difference between nfPPA patients and controls in this regard $[2,13,29]$. In contrast, Wilson et al. [23] found that their group of nfPPA patients produced ('marginally') fewer closed class words than controls, although it was noted that 9 of 14 patients were within the control range on this measure.

Many investigations of connected speech in nfPPA have documented reduction in grammatical complexity, which can occur in frank agrammatism as well as in less severe grammatical impairment. For example, studies have shown that nfPPA patients produce shorter utterances $[2,3,22-25,28-30]$, and fewer complex grammatical structures than controls [3, 23-25, 27-29, 31], but for a contrary result see Fraser et al. [26].

Thus, features of frank agrammatism are inconsistently documented in group studies of connected speech in nfPPA, and when documented there may be individuals whose results 
do not follow the group pattern. Clinical descriptions of speech in nfPPA also suggest that frank agrammatism is not a universal feature: Clark et al. [32] noted that only 3/21 of their nfPPA patients were frankly agrammatic, while Silveri et al. [33] indicated that $2 / 21 \mathrm{nfPPA}$ patients had 'agrammatic production', and Knibb et al. [34] reported that 13/23 exhibited 'dysgrammatism' in speech at presentation. Others have suggested that the grammatical impairment is not necessarily severe in nfPPA [23, 25, 29, 35].

The above review of the literature demonstrates that there is a high degree of variability across nfPPA patients with respect to grammatical skills. This could potentially be explained by at least four factors. First, it is possible that lvPPA patients have inadvertently been included in groups of nfPPA patients. Absence of frank agrammatism is a feature of lvPPA, and therefore inclusion of these patients could account for some of the diversity with respect to grammatical findings in nfPPA, particularly for studies published prior to the establishment of the consensus criteria [1]. A second (and related) possibility is that patients with nfPPA are classified as lvPPA because they do not exhibit frank agrammatism. The criteria acknowledge, however, that lack of frank agrammatism or AOS could arise in nfPPA, at least in the early stages of illness: 'Effortful speech and production errors can be the first symptoms of this variant, even before clear apraxia of speech or agrammatic errors occur' [1, p. 1010]. A third explanation for the variability in published results with respect to grammatical skills in nfPPA is that group studies may include variable numbers of patients whose dysfluency arises at least partially, or even primarily, from a motor speech impairment; these patients could have normal grammatical skills but still satisfy diagnostic criteria for nfPPA. Some researchers separate patients whose nonfluent progressive aphasia is associated with primarily grammatical or primarily motor speech impairment [e.g., 6, 36], but this can be problematic because both impairments may arise in the same patient. A fourth reason which could account for variability in findings with respect to grammatical impairment in nfPPA is stage of disease. It is possible that less grammatically impaired patients are at an early stage of illness and will inevitably develop more severe syntactic impairments and concomitant frank agrammatism.

In the present study, we addressed the question of whether frank agrammatism is typical in grammatically impaired nfPPA patients when this feature is not required for diagnosis. We systematically evaluated connected speech output in a group of patients with PPA with the aim of identifying frank agrammatism, when present. The patients were diagnosed clinically, and we used volumetric data from MRI to provide independent and unbiased support for the diagnosis. Because features of language output can overlap between the nonfluent and logopenic variants, it was important to determine whether the imaging findings could rule out lvPPA. We also assessed motor speech skills to evaluate the possibility that a motor speech impairment was either contributing to or primarily causing the dysfluency in any of the nfPPA patients. The present study had three aims: (1) to evaluate syntactic production in nfPPA; (2) to document our impression that frank agrammatism may not be universally present in nfPPA patients, even those with preserved motor speech skills, and (3) to address the question of diagnosis of agrammatic nfPPA and how it may be influenced by the definition of agrammatism used.

\section{Materials and Methods}

\section{Participants}

Data from 27 participants with a clinical diagnosis of PPA ( 9 nfPPA, 14 semantic variant (svPPA), 4 lvPPA) and 22 age- and education-matched healthy controls were collected during a longitudinal study of language impairments in PPA at the Department of Speech-Language Pathology, University of Toronto. Note that data from 7 of the $9 \mathrm{nfPPA}$ patients presented here 
were also reported in a previous study involving automated analysis of speech transcriptions in PPA [26]. Participants with PPA were recruited from four memory clinics in university hospitals. Although nfPPA is the disorder under study, patients with other PPA variants were included because their speech samples were used as distractors for the ratings of agrammatism and AOS, which were a major component of the investigation. Each patient was diagnosed by an experienced behavioral neurologist (S.B., D.F.T.-W., T.W.C., M.M.) on the basis of history, clinical features and MRI. Visual inspection of MRI scans was used for diagnostic purposes, but volumetric MRI data (reported below) were not. All patients diagnosed with the nonfluent variant had nonfluent, halting speech, but as documented below, not all exhibited frank agrammatism in production or AOS. Because we wanted to ensure that all of the nfPPA patients unequivocally met diagnostic criteria, we excluded those whose clinical diagnosis was not imaging supported, and those whose neuropsychological testing indicated that they did not meet two thirds of the subsidiary diagnostic criteria, as required for clinical diagnosis [1]. Each individual's conformity with subsidiary criteria is detailed below. Eight patients with a probable diagnosis of the nonfluent variant were excluded because they did not fulfill the requirements just described. Control participants were recruited from the Rotman Research Institute Participant Database.

All participants had to be able to complete neuropsychological testing in English, either as native speakers or having been formally educated in English, and all completed a standardized MRI research scan. Exclusion criteria included a known history of drug or alcohol abuse, or a history of neurological or major psychiatric illness. The data reported here are from the earliest of annual assessments for which there were both neuropsychological and imaging data available. For most participants, this was the first assessment, but a minority of patients ( $n=2$, cases 18 and 36$)$ were not scanned at their initial assessment because the scanner was out of commission for upgrading; for these participants, we used neuropsychological and imaging data from the second annual assessment. Demographic information for the participants is shown in table 1.

The research ethics boards of University of Toronto and the hospitals involved in recruitment (University Health Network, Sunnybrook Health Sciences Centre, Baycrest, and the Centre for Addiction and Mental Health) all approved the study. Written informed consent was obtained prior to the study from all participants.

\section{Background Neuropsychological Assessment}

All participants underwent neuropsychological testing, including assessment of language function (table 1). The omnibus group effects were significant for all neuropsychological and language tests, and post hoc tests showed that all patient groups were impaired relative to controls on most of the tests. Because this pattern of results gives the misleading impression that the patients have a generalized dementia, which would be inconsistent with a diagnosis of PPA, we also report the proportion of patients whose scores were within the normal range (i.e., the range of scores achieved by control participants). In general, these proportions reveal better preservation of nonverbal cognitive skills than is apparent from the group comparisons.

\section{Cognitive Assessment}

Overall cognitive function was assessed with the Mini-Mental State Examination [37]. All patient groups were impaired relative to controls on this test, and the majority of patients had abnormal scores. Because cognitive screening tests such as these rely considerably on language function, low scores were expected. Nonverbal reasoning was assessed with Raven's Coloured Progressive Matrices [38], and the majority of patients in all three groups achieved normal performance, although at the group level the nfPPA and IvPPA groups performed 
Dementia

Cognitive Disorders

$\cong$

$\stackrel{8}{9}$

ปัँ

ีㅗำ

$\frac{\pi}{2}$

공

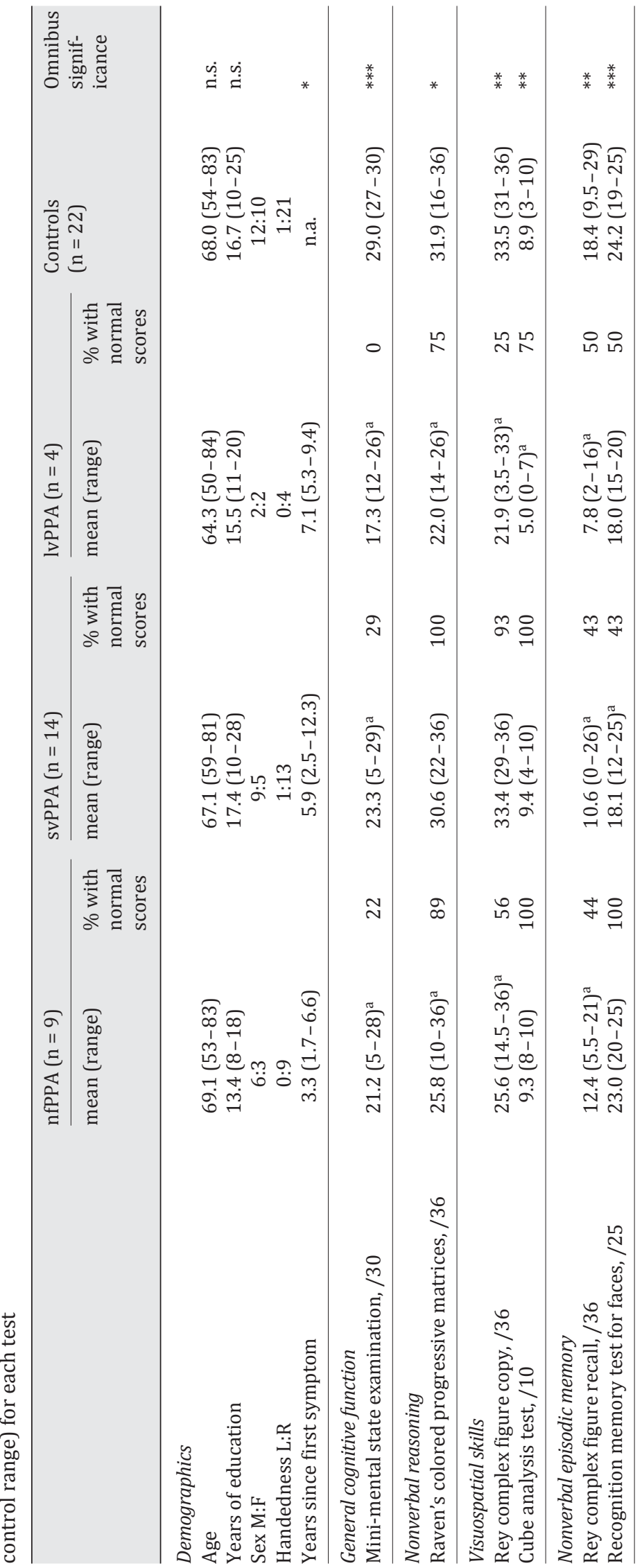

Graham et al.: Lack of Frank Agrammatism in the Nonfluent Agrammatic Variant of Primary Progressive Aphasia

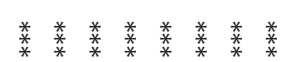<smiles>[CH]C=[AsH][AsH]</smiles>

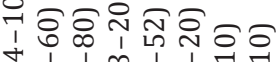
i 2 m $-7-6000$



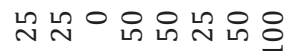



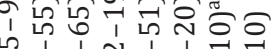

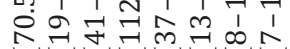
on 0 o m mo

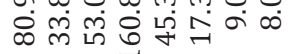

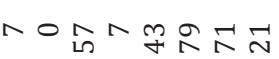

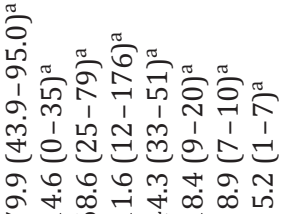



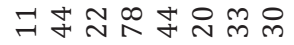

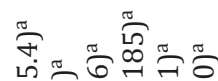

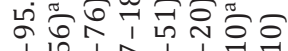

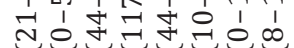

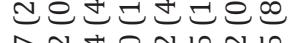

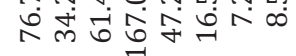

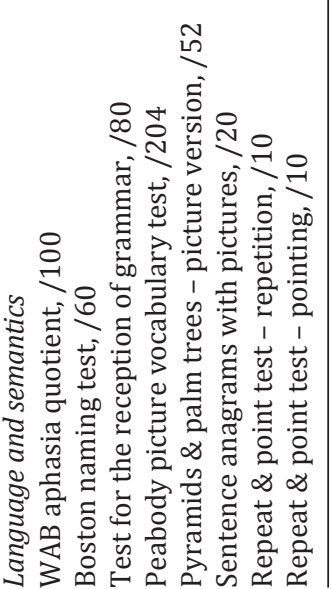


significantly worse than the control group. Visuospatial skills were assessed by having participants copy the Rey-Osterreith complex figure [39], and by the Cube Analysis Test [from the Visual Object \& Space Perception Battery, 40] which requires participants to count sets of cubes arranged in 3-dimensional space, but presented as 2-dimensional drawings. The nfPPA group was mildly impaired on copy of the complex figure, but showed normal performance on Cube Analysis. The svPPA group was not impaired on either of these tests, while the lvPPA group was impaired on both. Evaluation of episodic memory focused on nonverbal material. Participants were asked to recall the Rey-Osterreith complex figure $30 \mathrm{~min}$ after copying it, and to perform a forced-choice recognition test for 25 faces they had just been shown [41]. Each patient group was impaired relative to controls on recall of the complex figure, but the impairment was mild and all but one participant (with svPPA) scored above floor level. On recognition memory for faces, all of the nfPPA patients achieved scores in the normal range, while the svPPA and lvPPA groups were impaired.

\section{Language Assessment}

The aphasia quotient from the Western Aphasia Battery-Revised [42] provided a general indication of aphasia severity. There was a wide range of scores, particularly within the nfPPA group. Not surprisingly, all of the patient groups were impaired on this measure, and the scores of most individuals were below the normal range. Confrontation naming was assessed using the Boston Naming Test [43], and all of the patient groups and most of the individual patients were impaired, particularly the svPPA patients. The Test for the Reception of Grammar-2 [44] assesses comprehension of a range of simple and complex syntactic structures, and results indicated that syntactic comprehension was impaired in all of the patient groups, with only a minority of individuals achieving scores in the normal range. The nfPPA, svPPA, and lvPPA groups were also all impaired on single-word comprehension [Peabody Picture Vocabulary Test, 45]; at an individual level, 78\% of the nfPPA patients had normal scores, as did $50 \%$ of the lvPPA patients, but only one svPPA patient (i.e., $7 \%$ ) managed this, which is consistent with the semantic impairment in the latter group. On the Pyramids and Palm Trees Test [46], which is a test of nonverbal semantics involving conceptual matching of pictures, all of the groups were mildly impaired; at an individual level, approximately half of the patients achieved normal scores. We assessed sentence production using an anagram task [taken from the Verb and Sentence Test Battery, 47] in which segments of active or passive sentences were written on three cards and participants were required to arrange the cards to form a sentence which described a given picture. All of the patient groups were impaired relative to controls on the anagram task, and at an individual level, a greater proportion of svPPA than nfPPA or lvPPA patients had scores within the normal range. The final language test was the Repeat and Point Test [48], in which participants must repeat a multisyllabic word and then point to the corresponding picture in an array of seven pictures that are closely semantically related to the target. This test has been shown to distinguish nfPPA from svPPA [48] in that nfPPA patients have greater difficulty with the repetition task, while svPPA patients have greater difficulty with the pointing task, which is exactly what we found at both the group and individual levels.

\section{Neuropsychological Assessment - Characterization of Individual nfPPA Patients'}

Language Skills and Conformity with Subsidiary Diagnostic Criteria

We used the neuropsychological test scores to evaluate each nfPPA patient's concordance with the subsidiary features from the consensus criteria [1]. A diagnosis of nfPPA requires two of the following three subsidiary features: (1) impairment in comprehension of syntactically complex sentences, (2) preserved single-word comprehension, and (3) preserved object knowledge. The tests which respectively evaluated these domains were: Test for the 
Graham et al.: Lack of Frank Agrammatism in the Nonfluent Agrammatic Variant of Primary Progressive Aphasia

Table 2. Neuropsychological test results for individual nfPPA patients which are pertinent to diagnosis, as well as classification with respect to agrammatism and AOS

\begin{tabular}{|c|c|c|c|c|c|c|c|c|c|}
\hline \multirow[t]{2}{*}{$\begin{array}{l}\text { Patient/ } \\
\text { case No. }\end{array}$} & \multirow{2}{*}{$\begin{array}{l}\text { Length } \\
\text { of } \\
\text { history, } \\
\text { years }\end{array}$} & \multirow{2}{*}{$\begin{array}{l}\text { Frank } \\
\text { agram- } \\
\text { matism? }\end{array}$} & \multirow[t]{2}{*}{ AOS? } & \multirow{2}{*}{$\begin{array}{l}\text { WAB } \\
\text { aphasia } \\
\text { quotient, } \\
/ 100\end{array}$} & \multirow{2}{*}{$\begin{array}{l}\text { Boston } \\
\text { naming } \\
\text { test, / } 60\end{array}$} & \multicolumn{3}{|c|}{$\begin{array}{l}\text { Tests addressing subsidiary diagnostic criteria } \\
\text { for nfPPA }\end{array}$} & \multirow{2}{*}{$\begin{array}{l}\text { Number } \\
\text { of scores } \\
\text { meeting } \\
\text { subsidiary } \\
\text { criteria, /3 }\end{array}$} \\
\hline & & & & & & $\begin{array}{l}\text { Test for } \\
\text { Reception of } \\
\text { Grammar, /80 }\end{array}$ & $\begin{array}{l}\text { Peabody Picture } \\
\text { Vocabulary Test, } \\
\text { /204 }\end{array}$ & $\begin{array}{l}\text { Pyramids } \\
\text { and Palm } \\
\text { Trees, /52 }\end{array}$ & \\
\hline 44 & 2.3 & no & no & 95.4 & 56 & 76 & $184^{\mathrm{a}}$ & $51^{\mathrm{a}}$ & 2 \\
\hline 17 & 4.3 & no & no & 88.0 & 23 & 73 & $175^{\mathrm{a}}$ & 44 & $1^{b}$ \\
\hline 22 & 2.3 & no & no & 86.8 & 46 & $50^{\mathrm{a}}$ & $179^{\mathrm{a}}$ & 46 & 2 \\
\hline 31 & 1.7 & no & no & 86.4 & 45 & $44^{\mathrm{a}}$ & $176^{\mathrm{a}}$ & 46 & 2 \\
\hline 8 & 3.8 & no & no & 82.6 & 49 & $57^{\mathrm{a}}$ & $185^{\mathrm{a}}$ & $50^{\mathrm{a}}$ & 3 \\
\hline 32 & 2.1 & no & no & 81.4 & 36 & $64^{\mathrm{a}}$ & 142 & $48^{\mathrm{a}}$ & 2 \\
\hline 37 & 3.3 & no & no & 79.2 & 26 & $66^{\mathrm{a}}$ & $176^{\mathrm{a}}$ & 46 & 2 \\
\hline 36 & 6.6 & yes & yes & 69.4 & 27 & $61^{\mathrm{a}}$ & $169^{a}$ & 46 & 2 \\
\hline 18 & 3.9 & yes & yes & 21.0 & $0^{\mathrm{c}}$ & $46^{\mathrm{a}, \mathrm{d}}$ & 117 & $48^{\mathrm{a}}$ & 2 \\
\hline \multicolumn{4}{|c|}{ Control mean } & 99.1 & 55.1 & 78.1 & 193.6 & 50.8 & \\
\hline \multicolumn{4}{|c|}{ Control range } & $92.4-100$ & $42-60$ & $73-80$ & $163-201$ & $47-52$ & \\
\hline
\end{tabular}

For the purpose of characterizing each patient, length of history, WAB aphasia quotient and scores on the Boston Naming Test are included. Patients are ordered by their WAB aphasia quotient. For comparison, control means and ranges are included at the bottom of the table. ${ }^{\text {a }}$ core which meets a subsidiary diagnostic criterion for nfPPA (i.e., impaired syntactic comprehension, spared single-word comprehension, spared object knowledge). ${ }^{\mathrm{b}}$ At the time of this study, case 17 met only one of the subsidiary diagnostic criteria, but on follow-up syntactic comprehension had dropped into the impaired range, and the participant then met (the required) two subsidiary criteria. ${ }^{c}$ This patient scored 0 on spoken naming due to severe apraxia of speech. On written naming of the same items, she scored $33 / 60{ }^{d}$ This score is from a nonstandard test administration, in which stimulus sentences were presented in written (rather than spoken) format. The participant refused the standard method of test administration due to her severe comprehension deficit.

Reception of Grammar, Peabody Picture Vocabulary Test, and Pyramids and Palm Trees Test. Scores are listed in table 2, and those outside the normal range were deemed to be abnormal. The WAB aphasia quotient and Boston Naming Test are also included in table 2 to provide additional information on each individual's language skills.

All of the nfPPA patients fulfilled the requirement to meet two thirds of the subsidiary diagnostic criteria (table 2) because this was an inclusion criterion. At the time of this study, case 17 fulfilled only one subsidiary criterion, but on follow-up 1 year later two thirds of the subsidiary diagnostic criteria were fulfilled because syntactic comprehension had dropped into the impaired range, and we considered that diagnosis of the nonfluent variant was confirmed.

\section{Ratings of Agrammatism and AOS}

The speech of patients (but not controls) was rated for the presence of frank agrammatism by two experts in speech-language pathology (E.R., C.L.). In order to identify any patients whose dysfluency arose partially, or primarily, from a motor speech impairment, the raters also evaluated speech samples for AOS. The raters were blinded both to the variant of PPA with which an individual had been diagnosed and to the number of participants diagnosed with each variant. The raters independently evaluated speech samples (described below) in random order. Interrater agreement was high for both agrammatism (96\%) and AOS (96\%). Disagreement regarding diagnosis of frank agrammatism occurred with one of the nfPPA patients (case 31) and was resolved by listening to additional speech samples, to ascertain that the patient was not frankly agrammatic. Disagreement with respect to AOS occurred with a different nfPPA patient (case 44), although the raters did agree that speech 
Table 3. Checklists used to rate frank agrammatism and apraxia of speech



was dysarthric and that alternating and sequential motion rates were normal. To resolve the disagreement regarding AOS, the raters listened to the speech samples together and ultimately agreed there was no AOS.

\section{Evaluation of Agrammatism}

Samples of connected speech were collected using 'topic-directed interviews' [49]. Participants were asked five questions about topics that are familiar to them (e.g., their job or occupation, what they do each day, their family, etc.), and spoke uninterrupted until each response was completed. All interviews were video- and audio-recorded. The raters evaluated participants' speech guided by the checklist for frank agrammatism shown in table 3 [based upon 50]. The raters worked from recordings, but sample transcriptions are provided in the appendix.

\section{Evaluation of AOS}

Four speech samples from each patient were rated for AOS: (1) repetition of 10 words of increasing length (e.g., please, pleasing, pleasingly); (2) production of speech alternating and sequential motion rates (rapid repetition of puh-puh-puh..., tuh-tuh-tuh..., kuh-kuh-kuh..., puh-tuh-kuh); (3) repetition of polysyllabic words/phrases three times (e.g., 'harmonica', 'statistical analysis'), and (4) connected speech (as described above). The first three of these 
tasks were all adapted from Wertz et al. [51] and are described in greater detail by Duffy [52]. They were chosen because they are known to be sensitive to AOS. Speech was recorded using a digital voice recorder; additional use of video recordings for connected speech enabled evaluation of visual features of AOS such as articulatory groping.

All four speech samples for each participant were rated separately using an AOS checklist (table 3) which included abnormal features of prosody [based on 52] and of articulation [from the Apraxia Battery for Adults, ed 2, 53]. A decision regarding the presence of AOS was made for each sample separately. Subsequently, speech samples for each participant were considered together to determine whether the participant had AOS.

\section{MRI Acquisition and Analysis}

Structural magnetic resonance images, including a 3-D T1-weighted, interleaved proton density/T2-weighted and fluid attenuation inversion recovery (FLAIR) were acquired on a 3-tesla General Electric MR-750 (Milwaukee, Wis., USA) scanner at Sunnybrook Health Sciences Centre. Brain extraction and tissue segmentation were accomplished via a semiautomated procedure which provided volumetric information for 26 brain regions. Previous publications provide a detailed description of the procedure [54, 55], and the software is available for download at www.sabre.brainlab.ca. To account for both individual variation in brain/head size, and differing rates of atrophy progression between and within subjects, an atrophy score was calculated for each individual for each region: atrophy score $=$ tissue volume/lobar volume. Tissue volume is the sum of normal-appearing grey and white matter, and white matter hyperintensities. Lobar volume is the sum of tissue volume plus sulcal and ventricular CSF, and black holes (CSF filled spaces). Lower atrophy scores indicate greater atrophy.

Analysis focused on the areas specified for imaging-supported diagnosis of each variant of PPA [1]. The overlap between these brain areas and the regions provided by the semiautomated brain parcellation procedure is not exact, but the analysis still provides an objective evaluation of the correspondence between clinical diagnosis and area(s) of brain atrophy. The regions which were evaluated (and the variant expected to show predominant atrophy in each region) were: left inferior frontal (nfPPA), left insula (nfPPA), left anterior temporal (svPPA), left posterior temporal (lvPPA), and left inferior parietal (lvPPA).

\section{Results}

\section{Evaluation of Agrammatism}

Two of the 27 PPA patients had frank agrammatism (cases 18, 36), and as expected they were both from the nfPPA group (table 2). In one of these patients, the raters were unable to make the classification on the basis of the connected speech sample because speech output was so limited. For this case (18), the raters evaluated a topic-directed interview obtained one year prior to the study and concluded that there was frank agrammatism at that time. None of the remaining 7 nfPPA cases $(8,17,22,31,32,37,44)$, or any of the svPPA or lvPPA cases, were frankly agrammatic.

\section{Evaluation of AOS}

A total of 3 participants had AOS (table 2), including the 2 frankly agrammatic nfPPA cases $(18,36)$, and one svPPA patient. The remaining $7 \mathrm{nfPPA}(8,17,22,31,32,37,44)$ patients did not have AOS, although one had dysarthria (case 44, see below). In the svPPA patient with AOS, the AOS was mild and not apparent in connected speech; it was, however, noted in the production of speech sequential motion rates (i.e., accurate and quick repetition 
Table 4. Classification of individuals with nfPPA with respect to MRI volumetric data for key brain regions

\begin{tabular}{|c|c|c|c|c|c|c|c|c|c|c|}
\hline \multirow{2}{*}{$\begin{array}{l}\text { Interpreta- } \\
\text { tion of atro- } \\
\text { phy pattern }\end{array}$} & \multirow{2}{*}{$\begin{array}{l}\text { Patient/ } \\
\text { case No. }\end{array}$} & \multirow{2}{*}{$\begin{array}{l}\text { Length } \\
\text { of history, } \\
\text { years }\end{array}$} & \multirow{2}{*}{$\begin{array}{l}\text { WAB } \\
\text { aphasia } \\
\text { quotient, } \\
/ 100\end{array}$} & \multirow{2}{*}{$\begin{array}{l}\text { Frank } \\
\text { agramma- } \\
\text { tism? }\end{array}$} & \multirow[t]{2}{*}{ AOS? } & \multicolumn{5}{|c|}{ Brain region } \\
\hline & & & & & & $\begin{array}{l}\text { left } \\
\text { inferior } \\
\text { frontal }\end{array}$ & $\begin{array}{l}\text { left } \\
\text { insula }\end{array}$ & $\begin{array}{l}\text { left } \\
\text { anterior } \\
\text { temporal }^{\text {a }}\end{array}$ & $\begin{array}{l}\text { left } \\
\text { posterior } \\
\text { temporal }\end{array}$ & $\begin{array}{l}\text { left } \\
\text { inferior } \\
\text { parietal }\end{array}$ \\
\hline \multicolumn{11}{|c|}{ Imaging supported diagnosis of nfPPA $(n=6)$} \\
\hline & 44 & 2.3 & 95.4 & no & no & -1.68 & -3.27 & -1.88 & -0.98 & -1.30 \\
\hline & 17 & 4.3 & 88.0 & no & no & 0.48 & -2.03 & -2.15 & -1.44 & -1.31 \\
\hline & 31 & 1.7 & 86.4 & no & no & -3.01 & -3.47 & -4.94 & -1.57 & -1.75 \\
\hline & 8 & 3.8 & 82.6 & no & no & -2.48 & 0.32 & -1.22 & -0.72 & -0.53 \\
\hline & 32 & 2.1 & 81.4 & no & no & -2.03 & -3.48 & -8.58 & -1.45 & -0.03 \\
\hline & 18 & 3.9 & 21.0 & yes & yes & -3.69 & -3.15 & -2.47 & -1.78 & -1.12 \\
\hline & mean: & 3.5 & 78.5 & & & -2.12 & -2.53 & -4.18 & -1.42 & -1.00 \\
\hline \multicolumn{11}{|c|}{ Cannot rule out lvPPA on the basis of MRI data $(n=3)$} \\
\hline & 22 & 2.3 & 86.8 & no & no & -2.42 & -2.11 & -2.03 & -1.96 & -2.61 \\
\hline & 37 & 3.3 & 79.2 & no & no & 0.07 & -3.04 & -1.79 & -2.14 & -1.89 \\
\hline & 36 & 6.6 & 69.4 & yes & yes & -3.58 & -4.48 & -3.47 & -3.02 & -3.53 \\
\hline & mean: & 3.7 & 73.5 & & & -2.02 & -2.63 & -2.11 & -2.05 & -2.54 \\
\hline
\end{tabular}

Volumetric data are presented as z-scores and those which are considered abnormal appear in bold (i.e., z-scores below -2.0). Patients are ordered within each group by their WAB aphasia quotients. ${ }^{a}$ The significant left anterior temporal atrophy shown by some patients presumably arose because the area examined includes some of the anterior superior perisylvian region.

of puh-tuh-kuh). In addition, performance on the word repetition tasks was marked by sequencing difficulties and vowel distortions. This patient was not one of the more severe svPPA cases, scoring 23/30 on the MMSE and 21/60 on the Boston Naming Test, which suggests that the AOS did not arise as a concomitant of severe disease.

The raters also noted that 3 patients exhibited mild dysarthria, but without AOS. There was one dysarthric patient in each of the three patient groups, including case 44 from the nfPPA group. Although dysarthria is not common in the logopenic or semantic variants, it has been documented previously in both variants [56].

\section{Neuroimaging Results}

Analysis focused on atrophy scores for individual nfPPA patients (rather than group data) since a major motivation for examination of the MRI data was to rule out the possibility that the nfPPA group had inadvertently included lvPPA patients. For ease of interpretation, the atrophy scores have been converted to z-scores (based on control means and standard deviations). Atrophy scores were deemed to be abnormal if they were more than 2 standard deviations below the control mean.

Six $(67 \%)$ of the nfPPA patients $(8,17,18,31,32,44)$ had a pattern of atrophy which indicated that the clinical diagnosis was unequivocally imaging-supported (table 4). Their predominant atrophy was in the left inferior frontal and/or insular regions, and atrophy scores were normal for the posterior temporal and inferior parietal regions.

For the remaining $3(33 \%)$ nfPPA patients $(22,36,37)$, diagnosis of lvPPA could not be definitively ruled out on the basis of the MRI data (table 4). All of these patients (including one who had both frank agrammatism and AOS) had atrophy in the left posterior temporal and/or inferior parietal regions; they also had atrophy in the left insula, as expected in nfPPA. Although the imaging data from these three patients do not rule out lvPPA, they are compatible with a diagnosis of nfPPA. 


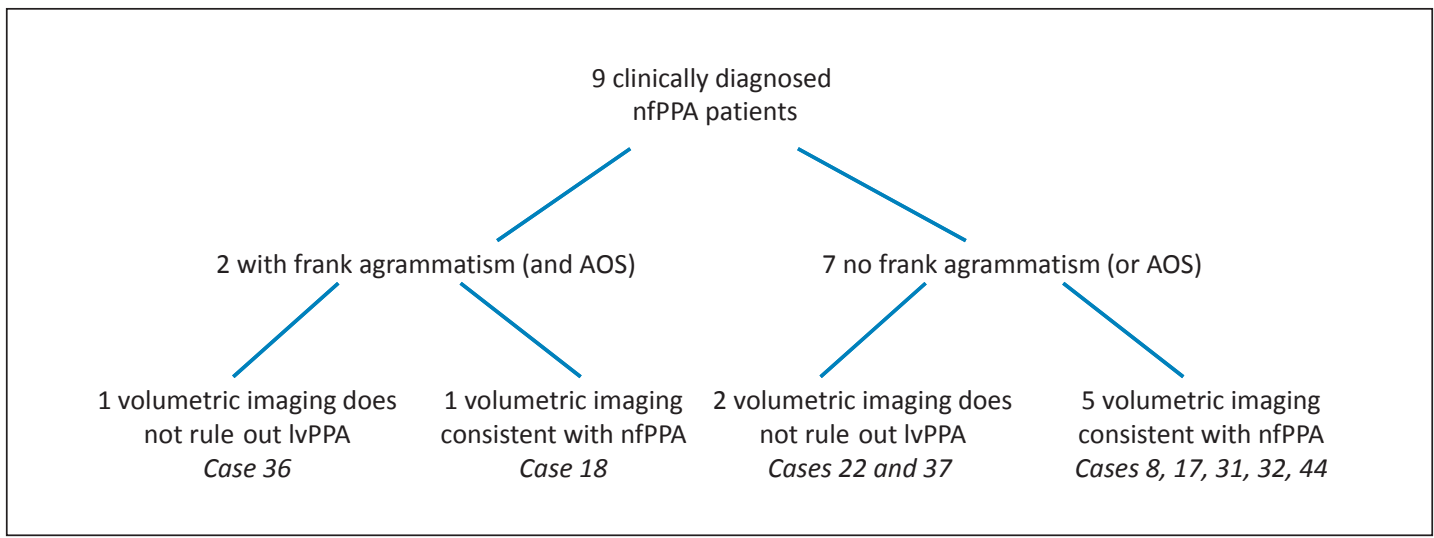

Fig. 1. Illustration of each nfPPA patient's results from the evaluations of agrammatism, AOS, and volumetric imaging data. Note that in this cohort, there did not happen to be any patients who had either frank agrammatism without AOS, or AOS without frank agrammatism, although these patterns of impairment would also fit within the spectrum of nfPPA.

The left anterior temporal region was included in the analysis as it is one of the areas specified for imaging-supported diagnosis of PPA. Although this region is primarily expected to be atrophic in SVPPA, 6 of the nfPPA patients had significant atrophy there. It is unlikely that a diagnosis of svPPA, or even 'mixed' PPA, could be applied to any of the participants with nfPPA because they all had nonfluent speech and did not show the degree of semantic impairment expected in svPPA. The significant left anterior temporal atrophy presumably arose because the area examined includes some of the anterior perisylvian region. Other reports have also noted left superior temporal atrophy in nfPPA $[22,57]$. We therefore did not take atrophy in this region to be mutually exclusive with imaging-supported diagnosis of nfPPA.

\section{Convergence of Study Measures on Diagnosis of nfPPA}

Figure 1 shows each nfPPA patient's results from the evaluation of agrammatism, together with their conformity with criteria for imaging-supported diagnosis based upon volumetric MRI data. Surprisingly, there was only 1 case (18) who unequivocally met the criteria for imaging-supported diagnosis and had frank agrammatism. The other patient who had frank agrammatism (case 36) did not clearly meet criteria for imaging-supported diagnosis of nfPPA (or for the other PPA variants) because the atrophy had spread beyond the left inferior frontal/insular regions to the posterior temporal and inferior parietal regions.

The remaining $7 \mathrm{nfPPA}$ patients did not exhibit frank agrammatism in connected speech. Although these patients were found to have preserved motor speech skills, they nevertheless had slow, effortful, nonfluent speech, and met core and subsidiary, criteria for clinical diagnosis. The analysis of volumetric MRI data demonstrated that $5(8,17,31,32,44)$ of these 7 cases unequivocally met criteria for imaging-supported diagnosis of nfPPA, rendering it unlikely that these are misdiagnosed lvPPA patients. For the remaining 2 patients $(22,37)$ who had neither frank agrammatism nor AOS, the clinical diagnosis of nfPPA was not clearly imaging-supported because there was atrophy in the left inferior parietal and/or posterior temporal regions; they also had atrophy in the left inferior frontal and/or insular regions (as expected in nfPPA).

It is important to question whether membership in the subgroups with versus without frank agrammatism is predicted in a straightforward manner by stage of illness. The numbers 
Graham et al.: Lack of Frank Agrammatism in the Nonfluent Agrammatic Variant of Primary Progressive Aphasia

were too small to perform meaningful analyses, but the length of history (at 3.9 and 6.6 years) tended to be longer in the 2 patients who had frank agrammatism than in the patients who did not (mean disease duration 2.8 years); there were, however, 2 patients in the latter group whose disease duration was as long as that of the one of the patients with frank agrammatism (case 8: 3.8 years; case 17: 4.3 years). Thus, some of the patients who did not have frank agrammatism were beyond the earliest stages of illness. It may be the case that patients with nfPPA will inevitably develop frank agrammatism (and/or AOS), but the present results demonstrate that agrammatism may remain mild, even beyond the earliest stages of the illness.

\section{Discussion}

The recent international consensus criteria for diagnosis of variants of PPA indicate that diagnosis of the nonfluent variant requires agrammatism in language production and/or AOS. In the literature on stroke-induced aphasia, the term 'agrammatism' is used to refer to speech characterized by omission and/or substitution of grammatical morphemes, with associated grammatical errors. In the literature on progressive aphasia, the term 'agrammatism' is used less consistently, and may refer to frank agrammatism (as just described) or to less severe grammatical impairment. In the series of nfPPA patients reported here, 'frank' agrammatism in production was not a necessary feature for diagnosis, although each patient did have nonfluent speech and met current diagnostic criteria [1]. We evaluated connected speech in 9 patients with clinically diagnosed nfPPA using blinded expert raters, and documented frank agrammatism in only a minority (2/9). Both of the frankly agrammatic cases exhibited AOS, but we could not discern any signs of an articulation planning deficit such as AOS in the remainder of the nfPPA patients, despite careful evaluation of four different speech samples (including three tasks known to be sensitive to AOS, as well as a sample of connected speech). Thus, the majority (7/9) of the nfPPA cases reported here had neither frank agrammatism nor AOS. This indicates that frank agrammatism is not universally present in nfPPA, even when motor speech impairment can be ruled out as contributing to or primarily causing the dysfluency.

One potential explanation for the finding that frank agrammatism occurred in only a minority of nfPPA patients is that patients with lvPPA, who are not expected to have frank agrammatism (or motor speech disorders), were inadvertently included in the nfPPA group. We investigated this possibility by using volumetric analysis of MRI data for brain regions expected to be atrophic in any variant of PPA. We documented 6 cases ( 5 of whom had neither frank agrammatism nor AOS) who unequivocally met criteria for imaging-supported diagnosis of nfPPA based on volumetric analysis of MRI data [1]: they had left inferior frontal and/ or left insular atrophy, as well as normal atrophy scores in the areas expected to be atrophic in lvPPA (left posterior temporal and left inferior parietal regions). This indicates that the lack of frank agrammatism observed in this subset of patients is unlikely to be due to erroneous inclusion of lvPPA patients in the group.

Two additional patients exhibited the same clinical syndrome (i.e., lack of frank agrammatism or AOS), but diagnosis of lvPPA could not be ruled out on the basis of the imaging data because these patients had left posterior temporal and/or inferior parietal atrophy (as well as the left insular atrophy excepted in nfPPA). In the literature, there are reports of nfPPA patients with left temporoparietal atrophy and of lvPPA patients with left inferior frontal atrophy [4, 58-61], but according to the criteria these should not be the predominant areas of atrophy in these variants. Thus, the imaging results showing co-occurrence of left inferior frontal and left temporoparietal atrophy are not incompatible with diagnosis of nfPPA, but 
neither do they allow us to distinguish definitively between nfPPA and lvPPA. The remaining nfPPA patient for whom diagnosis of lvPPA could not be ruled out on the basis of the imaging data (because there was temporoparietal atrophy) exhibited frank agrammatism, and thus unequivocally met clinical criteria for nfPPA and not lvPPA, despite not clearly meeting criteria for imaging-supported diagnosis of nfPPA.

We have shown that the lack of frank agrammatism in the majority of nfPPA patients reported here is unlikely to arise either from (legitimate) inclusion of patients whose dysfluency arises from motor speech impairment, or (for the majority of cases) from erroneous inclusion of lvPPA patients. Another potential explanation for the lack of frank agrammatism is that the patients were at an early stage of illness and will go on to develop this feature with disease progression. Some of our nfPPA patients who did not exhibit frank agrammatism or AOS were beyond the earliest stages of illness, but the idea that they will inevitably develop these features cannot be verified, or ruled out, without longitudinal followup. Although the majority of nfPPA patients reported here did not exhibit frank agrammatism in connected speech, we acknowledge that they may nevertheless have shown grammatical impairment on more difficult constrained production tasks [e.g., 13, 62, 63-65] or in writing $[27,66]$.

The present work demonstrates that patients with nfPPA, even those with preserved motor speech skills and imaging-supported diagnosis who are beyond the earliest stages of their illness, may not exhibit frank agrammatism. Although it is not widely recognized that nfPPA patients may have neither frank agrammatism nor AOS beyond the early stages of illness, it is consistent with findings in the literature (reviewed in the Introduction) indicating that features of frank agrammatism are not inevitably present in the speech of patients with nfPPA, and with the suggestion [67] that the language impairment in nfPPA does not necessarily parallel that seen in nonfluent agrammatic aphasia following stroke. When applying the diagnostic criteria, the term 'agrammatism' has been used inconsistently and sometimes frank agrammatism with syntactic errors is required for a diagnosis of nfPPA, particularly when patients are beyond the early stages of illness. If this type of language impairment is deemed essential for diagnosis of nfPPA, then the results of studies involving such patients will inevitably differ from studies involving patients (like the majority of those reported here) who have milder grammatical deficits. A separate but related issue is the differentiation of nfPPA and lvPPA. Because absence of frank agrammatism and absence of motor speech impairment are subsidiary criteria for diagnosis of lvPPA, some researchers classify as logopenic PPA patients whose speech is nonfluent but without frank agrammatism or AOS [e.g., $2,6]$. The present results demonstrate that if this approach were strictly applied, it could lead to misclassification in some cases.

With disease progression, the patients who have neither frank agrammatism nor AOS may go on to develop these features; alternatively, they may become progressively less fluent but maintain grammatical accuracy. The basis for the dysfluency presumably varies across patients, and this may account for the different patterns of language impairment reported here. Although the nature of the underlying impairment in nfPPA is not fully understood, within the literature, proposed causes include grammatical impairment, a planning disorder or impairment in executive function, and a motor speech disorder [5, 31, 33]; recently, it has been suggested that impairment in sensorimotor integration affecting processing of the individual's own speech input signal may also play a role [68].

Another implication of the present findings is that the spoken language impairments in nfPPA may be difficult to detect in the clinic because the grammatical impairment may be subtle, and not necessarily associated with obvious grammatical errors. Agrammatism is usually diagnosed by listening to patients' speech, rather than by quantitative analysis of output. Even when quantitative analyses show significant differences between nfPPA patients 
and controls on linguistic variables, the absolute numbers may be so small that the change may be difficult to detect clinically $[25,29]$. Some nfPPA patients produce numerous morphological and syntactic errors, and for these cases the diagnosis of agrammatism is indisputable, but we found that some nfPPA patients do not tend to make these errors. In these cases, the agrammatism may be made apparent by simplification of syntax and/or reduced utterance length $[23,29]$. Identification of these features may be difficult, and determination of whether there is a disease-related change in language may be complicated by individual variability in premorbid habits. In addition, simplification of syntax has been reported as a feature of speech in svPPA [69-71], and in dementia of the Alzheimer's type (DAT), even at the stage of mild cognitive impairment $[72,73]$. In contrast with nfPPA, however, the observed reduction in syntactic complexity in SVPPA and DAT was not associated with a reduction in speech fluency. Overall, the difficulty of detecting in the clinic language changes which are potentially subtle, demonstrates that further research on differentiation of speech impairment across variants is needed; in particular, we need a better understanding of the features that distinguish nfPPA from lvPPA, given that reduced speech fluency, lack of frank agrammatism and preserved motor speech skills can occur in both variants. Because the location of atrophy and the nature of the underlying impairment presumably differs across these variants, and possibly across patients who have the nonfluent variant, it should be possible to identify more specifically the unique features of the language impairments in these groups.

In conclusion, we have described a presentation of nfPPA that is consistent with diagnostic criteria but is not usually highlighted in the literature. The majority of the patients reported here had nonfluent speech but did not produce grammatical or articulatory errors. Longitudinal evaluation of language skills in additional nonfluent PPA patients, without necessitating frank agrammatism for diagnosis, is required to determine the clinical significance of these findings. In addition, further investigation is required to clarify the differences in the connected speech of patients with nfPPA but no frank agrammatism versus patients with lvPPA. Even if the lack of frank agrammatism occurs only in the early stages of illness in nfPPA, the present results suggest that this 'stage' can last for a number of years, and a better understanding of the differences between mild nfPPA and lvPPA could help with early differential diagnosis of the variants; this is an important goal because the likelihood of having different pathologies differs across variants [74], and treatment decisions may become pathology- and not symptom-based.

\section{Appendix}

Transcriptions of connected speech are provided below for 3 of the patients who met clinical and imaging criteria for nfPPA, but who did not exhibit frank agrammatism. These are taken from the speech samples evaluated by our expert raters. Because we used Topic Directed Interviews [49] in which participants are asked about themselves, most of the responses contained extensive identifying information. We have specifically chosen segments which do not contain this type of information; although they give a good indication of the grammatical structure of an individual's speech, the content is more general than is typical for these individuals. Numbers in brackets indicate the length of a pause for pauses lasting at least $1 \mathrm{~s}$.

\section{Patient 17}

Examiner: Tell me about what you do each day.

Patient: All right, uh, I get up (laughs), and uh, I keep a tidy house. My children can't believe why, how, but I, it doesn't get dirty, it doesn't do anything (laughs). So that's fine. It 
Graham et al.: Lack of Frank Agrammatism in the Nonfluent Agrammatic Variant of Primary Progressive Aphasia

doesn't, it's not too much work. But I read a lot, and uh, I look at television here and there, and uh (1) then, I do go out. I like to go and walk once a day, and uh I've just started again this week uh because I didn't uh walk. I don't like walking in the hot hot weather, and this is the nice kind of weather to walk. So I go out for my forty-five minute walk and (2), and I'll (1)... I don't do very much, really, uh, I go visiting and have an odd person in to have a cup of tea and uh, then on the weekends I go out with my family and (3) (patient nods and drinks some tea, indicating she has finished her response).

\section{Patient 32}

Examiner: Tell me about your health right now.

Patient: Well, obviously the um, physically I'm quite good but obviously this, the uh FTD is the problem. And mainly, I used to be very um eloquent, but now I'm struggling, with reading and hearing, um but still mathematics, 'cause I'm an XXX (states his occupation), so I was very fine. But as you, physically, well apparently, everything is fine, but not my brain.

\section{Patient 44}

Examiner: Tell me about what you do each day.

Patient: Hmm, well uh I golf uh probably three or four times a week. And then uh, maintain uh (2), the grass and the hedges and uh (2), there's uh, a lot of uh maintenance uh on the property. And uh, we uh socialize uh. We were at uh XXX's (name of a restaurant) uh last night with uh another couple. And uh, we closed the place (laughs).

\section{Acknowledgements}

This work was supported by the Canadian Institutes of Health Research (grant No. 82744). We thank Isabel Lam for assistance with study coordination and Amy Lewis, Shayna Sparling, Danna Rybko, Inderjit Sohal, and Ashleigh Wishen for help with testing participants. We especially thank the study participants and their families.

\section{References}

1 Gorno-Tempini ML, Hillis AE, Weintraub S, Kertesz A, Mendez M, Cappa SF, Ogar JM, Rohrer JD, Black S, Boeve BF, Manes F, Dronkers NF, Vandenberghe R, Rascovsky K, Patterson K, Miller BL, Knopman DS, Hodges JR, Mesulam MM, Grossman M: Classification of primary progressive aphasia and its variants. Neurology 2011; 76:1006-1014.

-2 Thompson CK, Cho S, Hsu C-J, Wieneke C, Rademaker A, Weitner BB, Mesulam MM, Weintraub S: Dissociations between fluency and agrammatism in primary progressive aphasia. Aphasiology 2012;26:20-43.

-3 Ash S, Evans E, O’Shea J, Powers J, Boller A, Weinberg D, Haley J, McMillan C, Irwin DJ, Rascovsky K, Grossman M: Differentiating primary progressive aphasias in a brief sample of connected speech. Neurology 2013;81: 329-336.

4 Mesulam MM, Wieneke C, Rogalski E, Cobia D, Thompson C, Weintraub S: Quantitative template for subtyping primary progressive aphasia. Arch Neurol 2009;66:1545-1551.

5 Grossman M: The non-fluent/agrammatic variant of primary progressive aphasia. Lancet Neurol 2012;11: 545-555.

6 Rohrer JD, Rossor MN, Warren JD: Syndromes of nonfluent primary progressive aphasia: a clinical and neurolinguistic analysis. Neurology 2010;75:603-610.

7 Mesulam MM, Wieneke C, Thompson C, Rogalski E, Weintraub S: Quantitative classification of primary progressive aphasia at early and mild impairment stages. Brain 2012;135:1537-1553.

-8 Thompson CK, Bastiaanse R: Introduction to agrammatism; in Bastiaanse R, Thompson CK (eds): Perspectives on Agrammatism. Hove, Psychology Press, 2012, pp 1-16.

-9 Saffran EM, Berndt RS, Schwartz MF: The quantitative analysis of agrammatic production - procedure and data. Brain Lang 1989;37:440-479. 
10 Wilson SM, Galantucci S, Tartaglia MC, Gorno-Tempini ML: The neural basis of syntactic deficits in primary progressive aphasia. Brain Lang 2012;122:190-198.

11 Blair M, Marczinski CA, Davis-Faroque N, Kertesz A: A longitudinal study of language decline in Alzhiemer's disease and frontotemporal dementia. JINS 2007;13:237-245.

12 Harciarek M, Sitek EJ, Kertesz A: The patterns of progression in primary progressive aphasia - implications for assessment and management. Aphasiology 2014;28:964-980.

13 Thompson CK, Meltzer-Asscher A, Cho S, Lee J, Wieneke C, Weintraub S, Mesulam M-M: Syntactic and morphosyntactic processing in stroke-induced and primary progressive aphasia. Behav Neurol 2013;26:35-54.

14 Thompson CK, Mack JE: Grammatical impairments in PPA. Aphasiology 2014;28:1018-1037.

15 Rohrer JD, Knight WD, Warren JE, Fox NC, Rossor MN, Warren JD: Word-finding difficulty: a clinical analysis of the progressive aphasias. Brain 2008;131:8-38.

16 Kertesz A, Harciarek M: Primary progressive aphasia. Scand J Psychol 2014;55:191-201.

17 Hodges JR, Patterson K: Nonfluent progressive aphasia and semantic dementia: a comparative neuropsychological study. J Int Neuropsychol Soc 1996;2:511-524.

18 Grossman M, Ash S: Primary progressive aphasia: a review. Neurocase 2004;10:3-18.

19 Grossman M, Mickanin J, Onishi K, Hughes E, D’Esposito M, Ding X-S, Alavi A, Reivich M: Progressive nonfluent aphasia: language, cognitive, and PET measures contrasted with probable Alzheimer's disease. J Cogn Neurosci 1996;8:135-154.

20 Mesulam MM: Primary progressive aphasia - a language-based dementia. N Engl J Med 2003;349:1535-1542.

21 Snowden JS, Neary D, Mann DMA: Fronto-Temporal Lobar Degeneration: Fronto-Temporal Dementia, Progressive Aphasia, Semantic Dementia. London, Churchill Livingstone, 1996.

-22 Grossman M, Powers J, Ash S, McMillan C, Burkholder L, Irwin D: Disruption of large-scale neural networks in non-fluent/agrammatic variant of primary progressive aphasia associated with frontotemporal degeneration pathology. Brain Lang 2013;127:106-120.

23 Wilson SM, Henry ML, Besbris M, Ogar JM, et al: Connected speech production in three variants of primary progressive aphasia. Brain 2010;133:2069-2088.

24 Ash S, McMillan C, Gunawardena D, Avants B, Morgan B, Khan A, Moore P, Gee J, Grossman M: Speech errors in progressive non-fluent aphasia. Brain Lang 2010;113:13-20.

25 Knibb JA, Woollams AM, Hodges JR, Patterson K: Making sense of progressive non-fluent aphasia: an analysis of conversational speech. Brain 2009;132:2734-2746.

26 Fraser KC, Meltzer JA, Graham NL, Leonard C, Hirst G, Black SE, Rochon E: Automated classification of primary progressive aphasia subtypes from narrative speech transcripts. Cortex 2014;55:43-60.

27 Graham NL, Patterson K, Hodges JR: When more yields less: speaking and writing deficits in nonfluent progressive aphasia. Neurocase 2004;10:141-155.

28 Ash S, Moore P, Vesely L, Gunawardena D, McMillan C, Anderson C, Avants B, Grossman M: Non-fluent speech in frontotemporal lobar degeneration. J Neurolinguistics 2009;22:370-383.

29 Sajjadi SA, Patterson K, Tomek M, Nestor PJ: Abnormalities of connected speech in non-semantic variants of primary progressive aphasia. Aphasiology 2012;26:1219-1237.

-30 Ash S, Moore P, Antani S, McCawley G, Work M, Grossman M: Trying to tell a tale: discourse impairments in progressive aphasia and frontotemporal dementia. Neurology 2006;66:1405-1413.

-31 Gunawardena BA, Ash S, McMillan C, Avants B, Gee J, Grossman M: Why are patients with progressive nonfluent aphasia nonfluent? Neurology 2010;75:588-594.

-32 Clark DG, Charuvastra A, Miller BL, Shapira JS, Mendez MF: Fluent versus nonfluent primary progressive aphasia: a comparison of clinical and functional neuroimaging features. Brain Lang 2005;94:54-60.

-33 Silveri MC, Pravata E, Brita AC, Improta E, Ciccarelli N, Rossi P, Colosimo C: Primary progressive aphasia: linguistic patterns and clinical variants. Brain Lang 2014;135:57-65.

34 Knibb JA, Xuereb JH, Patterson K, Hodges JR: Clinical and pathological characterization of progressive aphasia. Ann Neurol 2006;59:156-165.

-35 Auclair-Ouellet N: Inflectional morphology in primary progressive aphasia and Alzhiemer's disease: a systematic review. J Neurolinguist 2015;34:41-64.

-36 Josephs KA, Duffy JR, Strand EA, Machulda MM, Senjem ML, Lowe VJ, Jack CR Jr, Whitwell JL: Syndromes dominated by apraxia of speech show distinct characteristics from agrammatic PPA. Neurology 2013;81:337-345.

-37 Folstein MF, Folstein SE, McHugh PR: 'Mini-mental state'. A practical method for grading the cognitive state of patients for the clinician. J Psychiatr Res 1975;12:189-198.

38 Raven JC: Coloured Progressive Matrices Sets A, AB, B. London, HK Lewis, 1962.

39 Rey A: L'examen psychologique dans les cas d'encéphalopathie traumatique. Arch Psychol 1941;28:286-340.

40 Warrington EK, James M: The Visual Object and Space Perception Battery. Bury St Edmunds, Thames Valley Test Company, 1991.

41 Warrington EK: The Camden Memory Tests. Hove, Psychology Press, 1996.

42 Kertesz A: Western Aphasia Battery Revised. San Antonio, Harcourt Assessment, 2007.

43 Kaplan E, Goodglass H, Weintraub S: Boston Naming Test, ed 2. Philadelphia, Lippincott Williams \& Wilkins, 2001.

44 Bishop DVM: Test for the Reception of Grammar (TROG-2) Version 2. London, Psychological Corporation, 2003. 
45 Dunn LM, Dunn LM: Peabody Picture Vocabulary Test, ed 3. Circle Pines, American Guidance Service, 1997.

46 Howard D, Patterson K: Pyramids and Palm Trees: A Test of Semantic Access from Pictures and Words. Bury St Edmunds, Thames Valley Test Company, 1992.

47 Bastiaanse R, Edwards S, Rispens J: Verb and Sentence Test (VAST). San Antonio, PsychCorp, 2002.

48 Hodges JR, Martinos M, Woollams AM, Patterson K, Adlam AL: Repeat and point: differentiating semantic dementia from progressive non-fluent aphasia. Cortex 2008;44:1265-1270.

49 Orange JB, Kertesz A, Peacock J: Pragmatics in frontal lobe dementia and primary progressive aphasia. J Neurolinguistics 1998;11:153-177.

50 Saffran EM, Schwartz MF, Marin OSM: Evidence from aphasia: isolating the components of a production model; in Butterworth B (ed): Language Production. New York, Academic Press, 1980, vol 1, pp 221-241.

51 Wertz RT, LaPointe LL, Rosenbek JC: Apraxia of Speech in Adults: The Disorder and Its Management. New York, Grune\&Stratton, 1984.

52 Duffy JR: Motor Speech Disorders: Substrates, Differential Diagnosis and Management, ed 3. St. Louis, Elsevier/ Mosby, 2013.

53 Dabul B: Apraxia Battery for Adults, ed 2. Austin, PRO-ED, 2000.

54 Dade LA, Gao FQ, Kovacevic N, Roy P, Rockel C, O'Toole CM, Lobaugh NJ, Feinstein A, Levine B, Black SE: Semiautomatic brain region extraction: a method of parcellating brain regions from structural magnetic resonance images. Neuroimage 2004;22:1492-1502.

55 Ramirez J, Gibson E, Quddus A, Lobaugh NJ, Feinstein A, Levine B, Scott CJM, Levy-Cooperman N, Gao FQ, Black SE: Lesion explorer: a comprehensive segmentation and parcellation package to obtain regional volumetrics for subcortical hyperintensities and intracranial tissue. Neuroimage 2011;54:963-973.

56 Duffy JR, Strand EA, Josephs KA: Motor speech disorders associated with primary progressive aphasia. Aphasiology 2014;28:1004-1017.

57 Schroeter ML, Raczka K, Neumann J, Yves von Cramon D: Towards a nosology for frontotemporal lobar degenerations - a meta-analysis involving 267 subjects. Neuroimage 2007;36:497-510.

58 Rogalski E, Cobia D, Harrison TM, Wieneke C, Weintraub S, Mesulam M-M: Progression of language decline and cortical atrophy in subtypes of primary progressive aphasia. Neurology 2011;76:1804-1810.

59 Rogalski E, Cobia D, Martersteck A, Rademaker A, Wieneke C, Weintraub S, Mesulam M-M: Asymmetry of cortical decline in subtypes of primary progressive aphasia. Neurology 2014;83:1184-1191.

60 Rohrer JD, Caso F, Mahoney C, Henry M, et al: Patterns of longitudinal brain atrophy in the logopenic variant of primary progressive aphasia. Brain Lang 2013;127:121-126.

-61 Lu PH, Mendez MF, Lee GJ, Leow AD, Lee H-W, Shapira J, Jimenez E, Boeve BB, Caselli RJ, Graff-Radford NR, Jack CR, Kramer JH, Miller BL, Bartzokis G, Thompson PM, Knopman DS: Patterns of brain atrophy in clinical variants of frontotemporal lobar degeneration. Dement Geriatr Cogn Disord 2013;35:34-50.

-62 Weintraub S, Mesulam M-M, Wieneke C, Rademaker A, Rogalski EJ, Thompson CK: The Northwestern Anagram Test: measuring sentence production in primary progressive aphasia. Am J Alzheimers Dis Other Demen 2009; 24:408-416.

63 DeLeon J, Gesierich B, Besbris M, Ogar J, Henry ML, Miller BL, Gorno-Tempini ML, Wilson SM: Elicitation of specific syntactic structures in primary progressive aphasia. Brain Lang 2012;123:183-190.

64 Wilson SM, Brandt TH, Henry ML, Babiak M, Ogar JM, Salli C, Wilson L, Peralta K, Miller BL, Gorno-Tempini ML: Inflectional morphology in primary progressive aphasia: an elicited production study. Brain Lang 2014;136: 58-68.

65 Billette OV, Sajjadi SA, Patterson K, Nestor PJ: SECT and MAST: new tests to assess grammatical abilities in primary progressive aphasia. Aphasiology 2015;29:1135-1151.

66 Code C, Muller N, Tree JT, Ball MJ: Syntactic impairments can emerge later: progressive agrammatic agraphia and syntactic comprehension impairment. Aphasiology 2006;20:1035-1058.

67 Patterson K, Graham NL, Lambon Ralph MA, Hodges JR: Progressive non-fluent aphasia is not a progressive form of non-fluent (post-stroke) aphasia. Aphasiology 2006;20:1018-1034.

-68 Maruta C, Makhmood S, Downey LE, Golden HL, Fletcher PD, Witoonpanich P, Rohrer JD, Warren JD: Delayed auditory feedback simulates features of nonfluent primary progressive aphasia. J Neurol Sci 2015;347:345348.

69 Patterson K, MacDonald MC: Sweet nothings: narrative speech in semantic dementia; in Andrews S (ed): From Inkmarks to Ideas: Current Issues in Lexical Processing. Hove, Psychology Press, 2006, pp 299-317.

70 Sajjadi SA, Patterson K, Tomek M, Nestor PJ: Abnormalities of connected speech in semantic dementia. Aphasiology 2012;26:847-866.

71 Meteyard L, Quain E, Patterson K: Ever decreasing circles: speech production in semantic dementia. Cortex 2014;55:17-29.

-72 Ahmed S, Haigh AMF, de Jager CA, Garrard P: Connected speech as a marker of disease progression in autopsyproven Alzheimer's disease. Brain 2013;136:3727-3737.

73 Lyons K, Kemper S, LaBarge E, Ferraro FR, Balota D, Storandt M: Oral language and Alzheimer's disease: a reduction in syntactic complexity. Aging Cogn 1994;1:271-281.

74 Chare L, Hodges JR, Leyton CE, McGinley C, Tan RH, Kril JJ, Halliday GM: New criteria for frontotemporal dementia syndromes: clinical and pathological diagnostic implications. J Neurol Neurosurg Psychiatry 2014; 85:866-871. 